\title{
ARE LOCAL ACTIVE NETWORKS A RELIABLE DENSIFICATION OF NATIONAL RTK POSITIONING NETWORK? A CASE STUDY IN SPAIN
}

\author{
M $^{\text {a }}$ Selmira GARRIDO ${ }^{1)}$ *, Antonio J. GIL ${ }^{1)}$ and Rafael GAITÁN ${ }^{2)}$ \\ ${ }^{1)}$ Center for Advanced Studies in Earth Sciences. University of Jaén, Las Lagunillas Campus, 23071, Jaén, Spain \\ ${ }^{2}$ Accenture - Software Engineering, C/ Andalucia 15, P. 3, $7^{\circ} \mathrm{C}, 28760$, Tres Cantos, Madrid, Spain
}

*Corresponding author's e-mail: mgarrido@ujaen.es

\begin{tabular}{l} 
ARTICLE INFO \\
\hline Article history: \\
Received 11 November 2016 \\
Accepted 19 April 2017 \\
Available online 4 May \\
\hline
\end{tabular}

Keywords:

GNSS

RTK Positioning

Data acquisition

Quality control

RTK network

\begin{abstract}
The use of Continuously Operating Reference Stations, both singly and as part of an active network, is widely employed in surveying, engineering and other geomatics applications, achieving high accuracy positioning even in real time. With an active network the measurements of the reference stations are processed jointly in order to model the errors and compute network RTK corrections. Due to distance dependent errors (ionospheric and tropospheric delays), single base RTK positioning accuracy decreases with increased baseline length. However, the network solution (NRTK) retains the accuracy and the time to fix ambiguities (TTFA) at a constant level. This study aims to contribute to the scientific research on real time positioning based on active networks. In Southern Spain, ERGNSS, a national CORS network that provides GNSS data for post-processing and real time single-based reference station corrections, shares territory with the Andalusian Positioning Network (RAP), a local active network. RAP provides network and single-based RTK corrections. In order to analyze the quality of real time positioning based on both networks, several tests have been performed on a sample of test points. The reference frame, the time to fix ambiguity resolution, precision, accuracy and repeatability of RTK positioning are considered as the evaluation parameters. The results confirm that the RAP network complements the precise positioning services provided by the ERGNSS network, ensuring accurate real time positioning, full coverage and reliable positioning services in the Andalusian Community.
\end{abstract}

\section{INTRODUCTION}

Nowadays, Global Navigation Satellite Systems (GNSS) allow us to obtain positAions with high accuracy. These positions depend on the reference frame to which they are referred. Continuously Operating Reference Stations (CORS) collect and transmit the GNSS phase data to users and some stations are configured to stream error correction data to an internet server, allowing users to access the data through GSM/GPRS. CORS can be linked into networks to reduce errors and have the capability to deliver positioning services to a country, a state or even world-wide (Schwieger et al., 2009). The use of CORS, both singly and as part of a network (active network), is an option being used in surveying, engineering and other geomatics applications, achieving high positioning accuracy even in real time. The single baseline solution, also known as the single reference station (SRS) solution, uses one reference station (base station) to obtain correction data accessible either by radio transmission or via the internet. Common error sources associated with the use of a temporal base station, including inaccurate base coordinates, incorrectly plumbed base antenna and erroneous measurement of antenna height, are avoided by the use of a continuously operating reference station. With a CORS network the measurements of several reference stations are processed jointly in order to model the GNSS measurement errors and to compute network RTK corrections for the coverage area of the active network. While a sparse CORS network is sufficient for datum definition, the distance among permanent stations is limited to few tens of kilometers (50 $60 \mathrm{~km}$ ) to model the distance dependent errors. Wang et al. (2010) show that in areas where there are insufficient users to justify high densities of CORS stations, it may be possible to offer RTK services using more sparse networks.

The main advantages of an active network are: it requires a lower density of reference stations, it is more robust with respect to possible failures in a particular reference station, it improves work performance and it transmits a reference frame throughout the coverage area of the network. Different manufacturers provide slightly different concepts for network RTK corrections. The most widely used solutions are the FKP (Flächen-korrektur-parameter), the VRS (Virtual Reference Station) and the MAC (Master Auxiliary Concept) approach (Euler et al., 2001). Based on the latter, Leica Geosystems $(C)$ offers MAX and i-MAX solutions (Takac and Zelder, 2008). 
A master station is determined, typically the closest reference station to the rover and data from multiple reference stations are used to compute the network RTK corrections at the server and then broadcasted to the user in the case of i-MAX. An NMEA GGA string is required from the rover so that the software may choose the appropriate cell and interpolate the network corrections for the rover position. For the MAX solution the rover computes and applies the network corrections. An active network provides the information to the user for precise positioning (real time and post processing) in standard formats. For post-processing, the GNSS data are stored in RINEX (Receiver Independent Exchange) format. For data transmission in real time applications, the RTCM standard is used (Boriskin et al., 2012).

Today the number of national and regional active reference frames is very high. Many RTK networks are built and managed by Institutes of Geodesy and Cartography, Geographic Institutes and Scientific Organizations except in some cases where they are managed by survey engineering service providers. At the European level the EUREF-IP Project (Dettmering and Webber, 2004) is already consolidated. EPN stations streaming real-time data make their data available to the regional broadcasters, and if the station is part of a local network, e.g. ERGNSS in Spain, it can also make available the real-time data to a local broadcaster. The station position reported in the stream should be consistent with the ETRS89 coordinates at the current realization of this reference system. At national level, there are many active networks available in Europe. In Spain, ERGNSS network provides a free GNSS data for post processing and carrier phase differential corrections for RTK positioning (Spanish Committee of Geodesy and Geophysics, 2011). In Portugal, two nation-wide reference networks, RENEP (Rede Nacional de Estações Permanentes GNSS) managed by the Instituto Geografico Português and SERVIR (Sistema de Estações de Referência GNSS VIRtuais), an Army CORS Network (Afonso et al., 2008), coexist in order to support real-time high precision land surveying and other positioning applications. In Germany, the realtime service SAPOS HEPS was one of the first RTKservices using the Network RTK approach (Wegener and Wanninger, 2005). Other examples of active networks in Europe are found in Edwards et al. (2010), Tsakiri (2011) or (Bakula, 2013) to name a few. RTK networks complement the national geodetic infrastructure in these countries. The establishment and the management of active networks by government agencies and geographic institutes have evolved significantly in the last decade. According to the model shown in Rizos (2007) related to business and operational models for GNSS-RTK services, the trend is a government agency establishes a CORS network but licenses the raw CORS data (or the RTK messages) to one or more private service providers who undertake to market the services derived from the CORS data.
One aspect of particular relevance in an active network is to know the reference frame that is being transferred to users (Benciolini et al., 2008). The basis is a particular realization of the ITRS (International Terrestrial Reference System). It can be considered as being well-defined, long term stable, highly accurate and easily accessible, and it is the basis for precise positioning. The latest ITRS realization is the ITRF2014 published by the IERS on January 2016. For Europe, ETRS89 (European Terrestrial Reference System 1989), officially born in 1990, is coincident with ITRS at the epoch 1989.0 and fixed to the stable part of the Eurasian Plate. The EUREF Technical Working Group recommends expressing the ETRS89 coordinates in the ETRF2000, the conventional frame of the ETRS89 (Boucher and Altamimi, 2011). Spain introduced ETRS89 as national reference system in 2007 and simultaneously implemented the UTM (Universal Transverse Mercator) as a two-dimensional projection coordinate system.

The aim of this study is to analyze the quality of the real time positioning provided by a national and a local service available in Southern Spain (ERGNSS and RAP networks) and to contribute to the recent research on active networks.

\section{NATIONAL AND LOCAL ACTIVE NETWORKS IN SOUTHER SPAIN}

ERGNSS is a national CORS network managed by the Spanish National Geographic Institute since 1998; 75 stations form the network, 24 are integrated into the EUREF European Network and 3 (LPAL, MELI and YEBE) into the global network of the International GNSS Service (IGS). Several ERGNSS reference stations are located in the Andalusian Community (S Spain), sharing territory with the Andalusian Positioning Network, a local active network. Four stations, ALME (Almería), COBA (Córdoba), HUEL (Huelva) and MALA (Málaga) have been classified by EUREF as Class A stations (stations with positions at $1 \mathrm{~cm}$ precision and velocities at $1 \mathrm{~mm} / \mathrm{yr}$ precision at all epochs) and they can be used as fiduciary stations for EUREF densifications (Kenyeres, 2009). ERGNSS provides positioning services for high accuracy applications, including downloading of GNSS data (GPS+GLONASS) in compressed format and also provides RTK services, transmitting single-based reference station corrections in RTCM format using the NTRIP protocol (Network Transport of RTCM via Internet Protocol) (Weber et al., 2005). The ETRS89 coordinates for ERGNSS stations are available in ETRF05 (Brockmann, 2016) through FTP (ftp://ftp.geodesia.ign.es/ERGNSS). Table 1 shows ERGNSS reference stations located in the Andalusian Community, including their instrumentation and the GNSS data available.

The Andalusian Positioning Network (RAP are its initials in Spanish) is an active network located in Andalusia (Southern Spain) and managed by the Institute of Statistics and Cartography of Andalusia. 
Table 1 ERGNSS reference stations in the Andalusian Community (S Spain) (*Broadcaster: ergnss-ip.ign.es).

\begin{tabular}{|c|c|c|c|c|c|}
\hline $\begin{array}{l}\text { Id. Station } \\
\text { (IERS code) }\end{array}$ & $\begin{array}{c}\text { Installation } \\
\text { date }\end{array}$ & $\begin{array}{c}\text { Receiver } \\
\text { Antenna } \\
\text { (Dome) }\end{array}$ & Network & $\begin{array}{l}\text { Mount point (*)/ } \\
\text { RTCM Version }\end{array}$ & $\begin{array}{c}\text { Distance } \\
\text { to RAP } \\
\text { Nearest station }(\mathrm{km}) \\
\end{array}$ \\
\hline $\begin{array}{c}\text { ALME } \\
(13437 \mathrm{M} 001)\end{array}$ & $\begin{array}{c}\text { December } \\
1999\end{array}$ & $\begin{array}{c}\text { TRIMBLE NETRS } \\
\text { TRM29659.00 } \\
\text { (NONE) }\end{array}$ & $\begin{array}{c}\text { EPN } \\
\text { ERGNSS }\end{array}$ & ALME0 / 2.3 & 2.0 \\
\hline $\begin{array}{c}\text { COBA } \\
(13453 \mathrm{M} 001)\end{array}$ & $\begin{array}{l}\text { April } \\
2004\end{array}$ & $\begin{array}{l}\text { LEICAGRX1200 } \\
\text { LEIAR20 } \\
\text { (LEIM) }\end{array}$ & $\begin{array}{c}\text { EPN } \\
\text { ERGNSS }\end{array}$ & $\begin{array}{l}\text { COBA1 / } 2.3 \\
\text { COBA0 / } 3.1\end{array}$ & 7.2 \\
\hline $\begin{array}{c}\text { HUEL } \\
\text { (13451M001) }\end{array}$ & $\begin{array}{l}\text { December } \\
2001\end{array}$ & $\begin{array}{c}\text { TRIMBLE NETR9 } \\
\text { TRM29659.00 } \\
\text { (NONE) }\end{array}$ & $\begin{array}{c}\text { EPN } \\
\text { ERGNSS }\end{array}$ & $\begin{array}{l}\text { HUEL1 / } 2.3 \\
\text { HUEL0 / } 3.1\end{array}$ & 8.9 \\
\hline $\begin{array}{c}\text { MALA } \\
(13443 \mathrm{M} 001)\end{array}$ & $\begin{array}{l}\text { March } \\
2000\end{array}$ & $\begin{array}{l}\text { LEICA GR25 } \\
\text { LEIAR25.R4 } \\
\text { (LEIT) }\end{array}$ & $\begin{array}{c}\text { EPN } \\
\text { ERGNSS }\end{array}$ & $\begin{array}{l}\text { MALA1 / } 2.3 \\
\text { MALA0 / } 3.1\end{array}$ & 3.9 \\
\hline $\begin{array}{c}\text { TARI } \\
\text { (19350M001) }\end{array}$ & $\begin{array}{l}\text { May } \\
2010\end{array}$ & $\begin{array}{l}\text { LEICA GR25 } \\
\text { LEIAR20 } \\
\text { (LEIM) }\end{array}$ & ERGNSS & $\begin{array}{l}\text { TARI1 / } 2.3 \\
\text { TARI0 / } 3.1\end{array}$ & 18.3 \\
\hline
\end{tabular}

This network provides GPS observations for postprocessing applications in compressed RINEX format with adjustable recording intervals and time periods, and real-time differential corrections for accurate positioning (Páez et al., 2017). This network comprises 22 reference stations (10 first level and 12 second level stations) with mean inter-station distances of $70 \mathrm{~km}$ over a region of $87.268 \mathrm{~km}^{2}$. The installation of each reference station includes a dual frequency GNSS receiver from Leica Geosystems $\odot$. Specifically, GRX1230 Pro receiver, LEIAT504 (LEIS) antenna, weather station and two radiomodems for broadcasting the RTK corrections via radio transmission from first level stations. The second level stations have GRX1200 Pro receivers and LEIAX1202 (NONE) antenna. Leica SpiderNet software produces the network corrections and broadcast to the RAP's users over the Internet in standard RTCM format using the NTRIP protocol.

The RAP Network provides MAX and i-MAX network corrections based on the Master Auxiliary Concept (MAC) and single-based corrections. In this case, the user can choose the nearest single base solution or a specific reference station selecting the required "mount point". The network (NRTK) corrections available are in RTCM 3.1 format, including messages: 1004, 1006, 1008, 1014, 1015, 1016 and 1033 for MAX solution and messages: 1004, 1006, 1008, 1032 and 1033 for i-MAX solution. The single-based corrections are in RTCM 2.3 format, including messages: 1, 2, 3, 20, 21, 22 and 24 considering the nearest reference station (NRS) solution or the solution for a specific reference station (SRS). The RAP, as active network, realizes a reference frame and propagates it to the users and needs to be linked to the available terrestrial reference frames. The final coordinates of RAP reference stations were computed in ITRF05 considering Spanish and Portuguese IGS/EUREF GNSS reference stations, and transformed into the ETRS89 (ETRF05).

\section{QUALITY CONTROL METHODOLOGY FOR REAL TIME POSITIONING}

In order to analyze the quality of real time positioning based on ERGNSS and RAP networks, the availability of centimetric real time positioning, the time to fix ambiguity resolution (TTFA), the precision and accuracy and the repeatability of RTK positioning are considered as the evaluation parameters. The time to fix ambiguity resolution is the observation time required to resolve integer ambiguities in real-time after re-initialization. Generally, in NRTK positioning the TTFA is independent of the distance to the reference station and kept at a constant level lower than 90 seconds (Seeber, 2003). Precision is given in terms of standard deviation, and the repeatability of NRTK solutions is analyzed by considering multiple observation sessions at each test point. Accuracy is defined by Feng and Wang (2008) as the degree of conformance of an estimated position at a given time to a defined reference value ('true' value) which is obtained from an independent approach at a higher level of accuracy. In this study, the post-processing solution computed will be considered as "true value".

\subsection{EQUIPMENT CONFIGURATION AND DATA ACQUISITION AT TEST POINTS}

The test points selected are geodetic sites belonging to a national passive network supported by the Spanish National Geographic Institute. Each geodetic site is composed of a concrete cylinder $(1.20 \mathrm{~m}$ in height and $0.30 \mathrm{~m}$ in diameter) supported on $1 \mathrm{~m} \mathrm{x} 1 \mathrm{~m}$ base, with a reinforced centre to ensure 


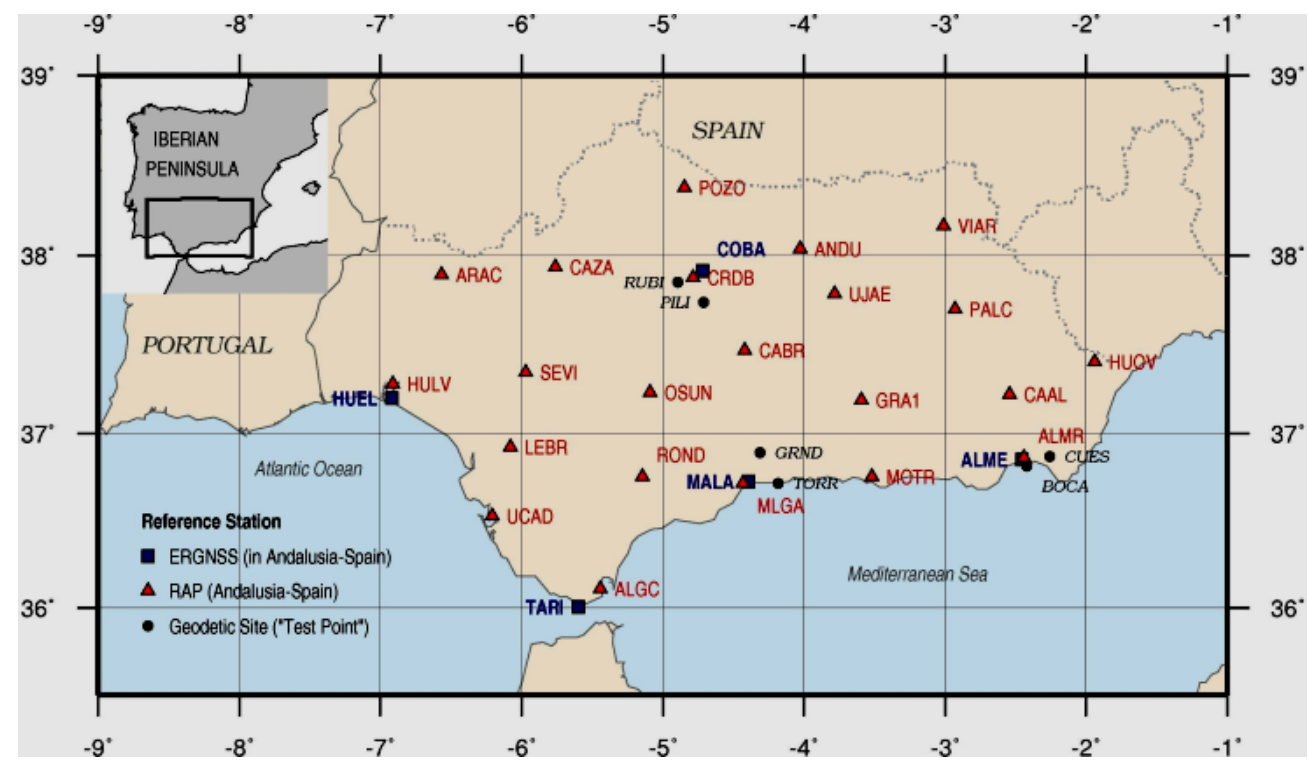

Fig. 1 Distribution of ERGNSS and RAP reference stations and test points in Southern Spain.

Table 2 Geodetic sites selected as test points, including geodetic coordinates (ETRS89) provided by the Spanish National Geographic Institute (IGNE), GPS week and day, baseline length (D) and elevation difference $(\Delta \mathrm{h})$ to the ERGNSS and RAP nearest reference stations.

\begin{tabular}{|c|c|c|c|c|c|c|c|}
\hline $\begin{array}{c}\text { Id. } \\
\text { Test Point }\end{array}$ & Longitude (W) & Latitude (N) & h (m) & $\begin{array}{c}\text { GPS week/ } \\
\text { day }\end{array}$ & $\begin{array}{c}\text { Id. } \\
\text { Nearest Station } \\
\end{array}$ & D (m) & $\Delta \mathbf{h}(\mathbf{m})$ \\
\hline \multirow{2}{*}{ BOCA } & \multirow{2}{*}{$2^{\circ} 25^{\prime} 35.02213^{\prime \prime}$} & \multirow{2}{*}{$36^{\circ} 48^{\prime} 41.15380^{\prime \prime}$} & \multirow{2}{*}{56.938} & $1646 /$ & ALME (ERGNSS) & 5431.138 & 69.579 \\
\hline & & & & 207 & ALMR (RAP) & 5830.770 & 140.837 \\
\hline \multirow{2}{*}{ CUES } & \multirow{2}{*}{$2^{\circ} 15^{\prime} 44.30869^{\prime}$} & \multirow{2}{*}{$36^{\circ} 52^{\prime} 02.50773^{\prime}$} & \multirow{2}{*}{198.623} & $1646 /$ & ALME (ERGNSS) & 17656.927 & -71.106 \\
\hline & & & & 209 & ALMR (RAP) & 15931.600 & 0.152 \\
\hline \multirow{2}{*}{ PILI } & \multirow{2}{*}{$4^{\circ} 42^{\prime} 52.54798^{\prime}}$, & \multirow{2}{*}{$37^{\circ} 44^{\prime} 23.64139^{\prime \prime}$} & \multirow{2}{*}{411.368} & $1644 /$ & COBA (ERGNSS) & 19510.396 & -209.286 \\
\hline & & & & 191 & CRDB (RAP) & 16568.213 & -215.345 \\
\hline \multirow{2}{*}{ RUBI } & \multirow{2}{*}{$4^{\circ} 53^{\prime} 42.63236^{\prime \prime}$} & \multirow{2}{*}{$37^{\circ} 51^{\prime} 10.06320^{\prime \prime}$} & \multirow{2}{*}{156.300} & $1643 /$ & COBA (ERGNSS) & 16824.827 & 45.782 \\
\hline & & & & 190 & CRDB (RAP) & 9832.145 & 39.723 \\
\hline \multirow{2}{*}{ GRND } & \multirow{2}{*}{$4^{\circ} 18^{\prime} 36.80451^{\prime \prime}$} & \multirow{2}{*}{$36^{\circ} 53^{\prime} 26.94849^{\prime}$} & \multirow{2}{*}{881.423} & $1645 /$ & MALA (ERGNSS) & 19732.142 & -761.576 \\
\hline & & & & 201 & MLGA (RAP) & 22425.871 & -765.748 \\
\hline \multirow{2}{*}{ TORR } & \multirow{2}{*}{$4^{\circ} 11^{\prime} 12.61913^{\prime \prime}$} & \multirow{2}{*}{$36^{\circ} 42^{\prime} 57.63651^{\prime \prime}$} & \multirow{2}{*}{89.352} & $1645 /$ & MALA (ERGNSS) & 18499.179 & 30.496 \\
\hline & & & & 200 & MLGA (RAP) & 22207.881 & 26.323 \\
\hline
\end{tabular}

an accurate and stable placement. Six test points were selected within a $20 \mathrm{~km}$ radius of each ERGNSS station considered. These ERGNSS stations were ALME, COBA and MALA due to thein proximity to a RAP station (Fig. 1). The test sites selected are summarized in Table 2, including their geodetic coordinates (ETRS89) provided by the IGNE, distance and elevation difference from the ERGNSS and RAP nearest reference stations. Test points and ERGNSS and RAP reference stations in the analysis region are shown in Figure 1.

At each test point two GNSS receiver Leica GX1230+ were connected to the same multi GNSS compact antenna with built-in ground plane Leica AS10 via a double-output amplified GPS source splitter (Fig. 2). Receiver $n^{0} 1$ was configured for a session of static measurements and 10 hours of raw 1 -second GNSS data were collected. Receiver $n^{\circ} 2$ was equipped with a Siemens MC75 GSM/GPRS module to receive RTK corrections from the ERGNSS and RAP NTRIP servers. The surveying equipment requires a single operator. The tests applied consider the network (NRTK: i-MAX solution) and nearest reference station (NRS) solutions for the RAP network and single reference station (SRS) for the ERGNSS network. In all of them the RTK configuration and setting were: 1-second observation rate, maximum PDOP 6 and measurement number 1 . An RTK solution is stored in "automatic mode" only if the TTFA $<90 \mathrm{~s}$ and the horizontal and vertical coordinate quality (CQ) is within the limits: CQ2D < $0.030 \mathrm{~m}$ and CQ1D $<0.050 \mathrm{~m}$. The RTK solution is stored in "manual mode" if the TTFA $=90$ s and is considered "failed" if the TTFA > 90s. The RTK tests were performed along three observation sessions each day (S1: morning; S2: mid-day and S3: afternoon). Each session had a sample size of 30 independent RTK positions. In order to reduce the correlation between two consecutive RTK positions, after recording each position the receiver was disconnected 


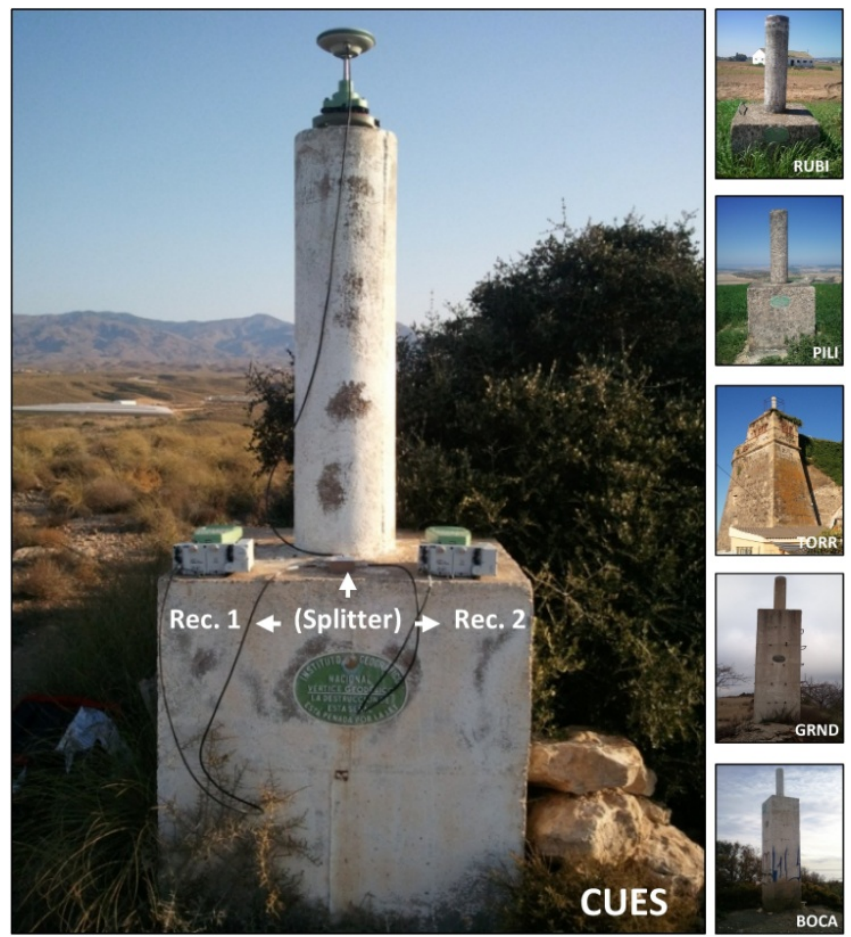

Fig. 2 Monumentation of geodetic sites selected as test points including the equipment connection for the GNSS data collection at "CUES" test point.

from the NTRIP caster and connected again when the phase solution was lost, ensuring independence between different ambiguities resolutions.

\subsection{DATA PROCESSING}

GNSS observations collected simultaneously to the RTK tests are post-processed relative to the ERGNSS and RAP reference stations in order to ensure that the control coordinates for each test site and each RTK position are in a compatible realization of the European Terrestrial Reference System (ETRS89). The GNSS static observation was postprocessed relative to the nearest ERGNSS reference station and the GPS observation was post-processed and adjusted relative to the three nearest RAP reference stations. The carrier phase observables were processed in a single baseline mode using GPS and GLONASS precise ephemeris and the antenna phase center model. $15^{\circ}$ elevation cut off angle was used. The discrepancies in the East, North and Up components obtained upon comparing the ETRS89based coordinates, determined with respect to the ERGNSS and RAP reference frames, are listed in Table 3, considering the ERGNSS solution as origin. Discrepancies in the East and North components were less than one centimeter. These discrepancies increased significantly for the Up component. All except one were less than four centimeters, reaching $77 \mathrm{~mm}$ at the GRND test point. Although both networks have the same reference system (ETRS89) and were computed with respect to the same reference frame (ETRF2005), the mean observation epochs are different, that is 2007.14 for the RAP network and 2007.81 for the ERGNSS network. At the GRND test point, if we compare the two solutions estimated with the ETRS89 solution offered by the Spanish National Geographic Institute (IGNE) shown in Table 2, we check that the altimetric bias is due to the ERGNSS solution.

Considering the IGNE solution as origin, the discrepancy in the Up component is $-0.085 \mathrm{~m}$ for the ERGNSS solution, but only $-0.008 \mathrm{~m}$ for the RAP solution. The last solution was derived from a leastsquares adjustment, while the ERGNSS solution was computed with respect to a single reference station and was affected by the length of the baseline and the large elevation difference between the reference station and the GRND test site (Table 2). As shows Bruyninx (2007), adding GLONASS data to GPS, the coordinates can change at the level of $1-2 \mathrm{~mm}$ in the horizontal components and between 2 and $6 \mathrm{~mm}$ for the vertical component. For the horizontal

Table 3 Discrepancies in the local geodetic frame (East, North and Up components) by comparing ERGNSS and RAP postprocessing solutions in ETRS89.

\begin{tabular}{lccc}
\hline Id. Test Point & East $(\mathbf{m})$ & North $(\mathbf{m})$ & Up $(\mathbf{m})$ \\
\hline BOCA & 0.001 & -0.002 & -0.019 \\
CUES & 0.001 & -0.006 & 0.030 \\
PILI & 0.002 & -0.007 & -0.020 \\
RUBI & 0.010 & 0.007 & 0.038 \\
GRND & -0.009 & -0.001 & 0.077 \\
TORR & 0.013 & -0.007 & -0.005 \\
\hline
\end{tabular}




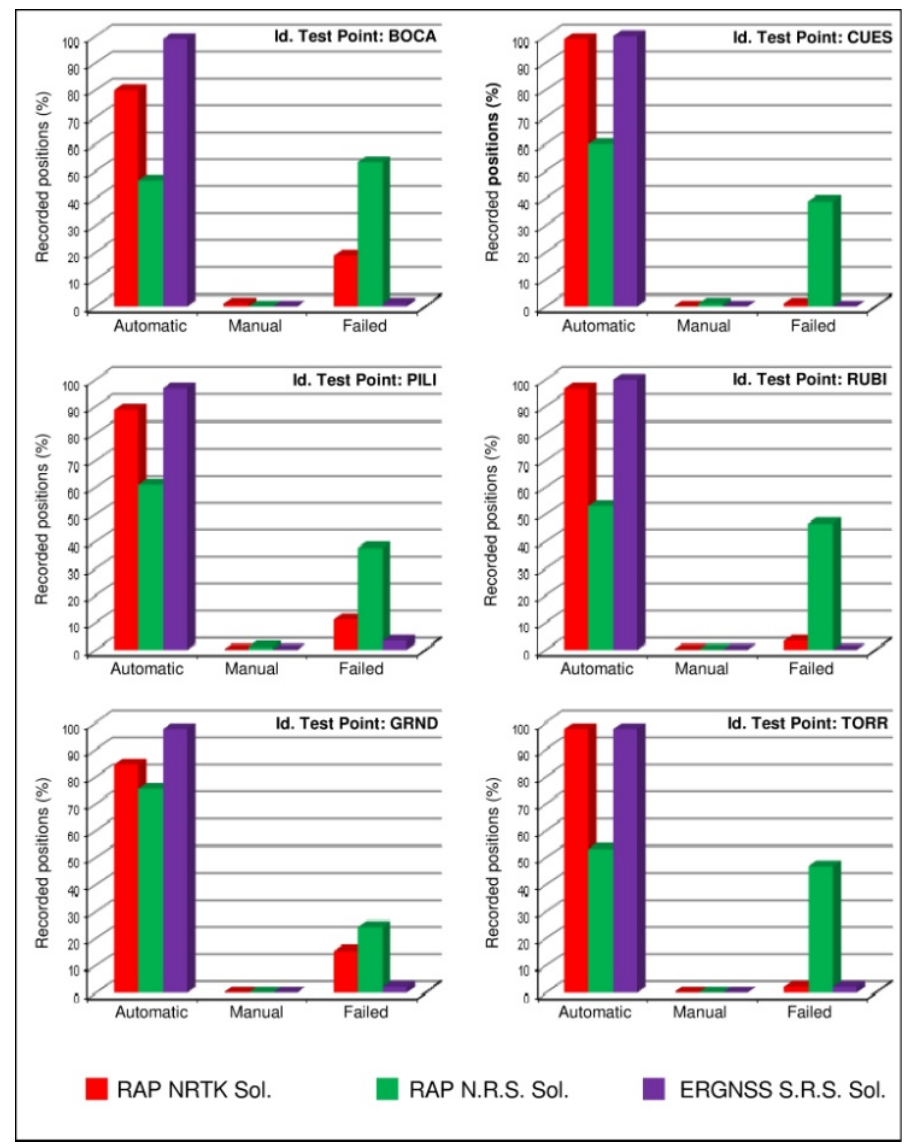

Fig. 3 Percentage of registered positions and failed ones considering the TTFA for the three RTK solutions considered.

components, the coordinate differences are mainly caused by reference frame differences between the two regional networks. The cause of this difference in the Up component may be due to the effect of the Troposphere. As it is strongly correlated with the altitude (Lei et al., 2010), the tropospheric delay predominately affects the vertical component. For short baselines with small elevation differences, the tropospheric delay is very similar on both ends of a baseline and the corresponding bias can be reduced by double differencing of the observations.

\section{RESULTS OF PRECISE REAL TIME POSITIONING}

This section provides numerical and graphical results of RTK positioning in order to analyze the evaluation parameters described in section 3 . In order to fix the phase ambiguities at least the same five satellites should be observed at the master station and the rover position. A low number of satellites available can produce high HDOP (horizontal dilution of precision) and VDOP (vertical dilution of precision) which decreases the accuracy of RTK positioning and even make it unable to reach a real time solution. Telecommunication coverage and latency corrections are other critical factors in RTK positioning. Figure 3 shown the percentages of records whose time to fix ambiguities is less than, equal to, or greater than 90 seconds at six test points analyzed.

ERGNSS S.R.S and RAP NRTK solutions follow a similar trend. If the ERGNSS S.R.S solution is considered, we can see that in all test points the percentage of registered positions in automatic mode is greater than $97 \%$. No positions are recorded in manual mode. For the RAP NRTK solution, the percentage of registered positions in automatic mode is between $80 \%$ and $99 \%$. These values correspond to BOCA and CUES test points respectively. This percentage is reduced for the RAP N.R.S solution, with values that oscillate between $47 \%$ (BOCA) and $76 \%$ (GRND). The lowest percentage of registered positions whose time to fix ambiguities is less than 90 seconds is concentrated at the BOCA test point for the RAP solutions, although this point is the closest to the reference station as shown in Table 2, it is located on the southern border of Andalusia and the NRTK solution is extrapolated.

Another objective of this study is to analyze the effects of the distance to the nearest reference station on the results of precision in RTK positioning. For short baseline, signal paths from the satellite to both 


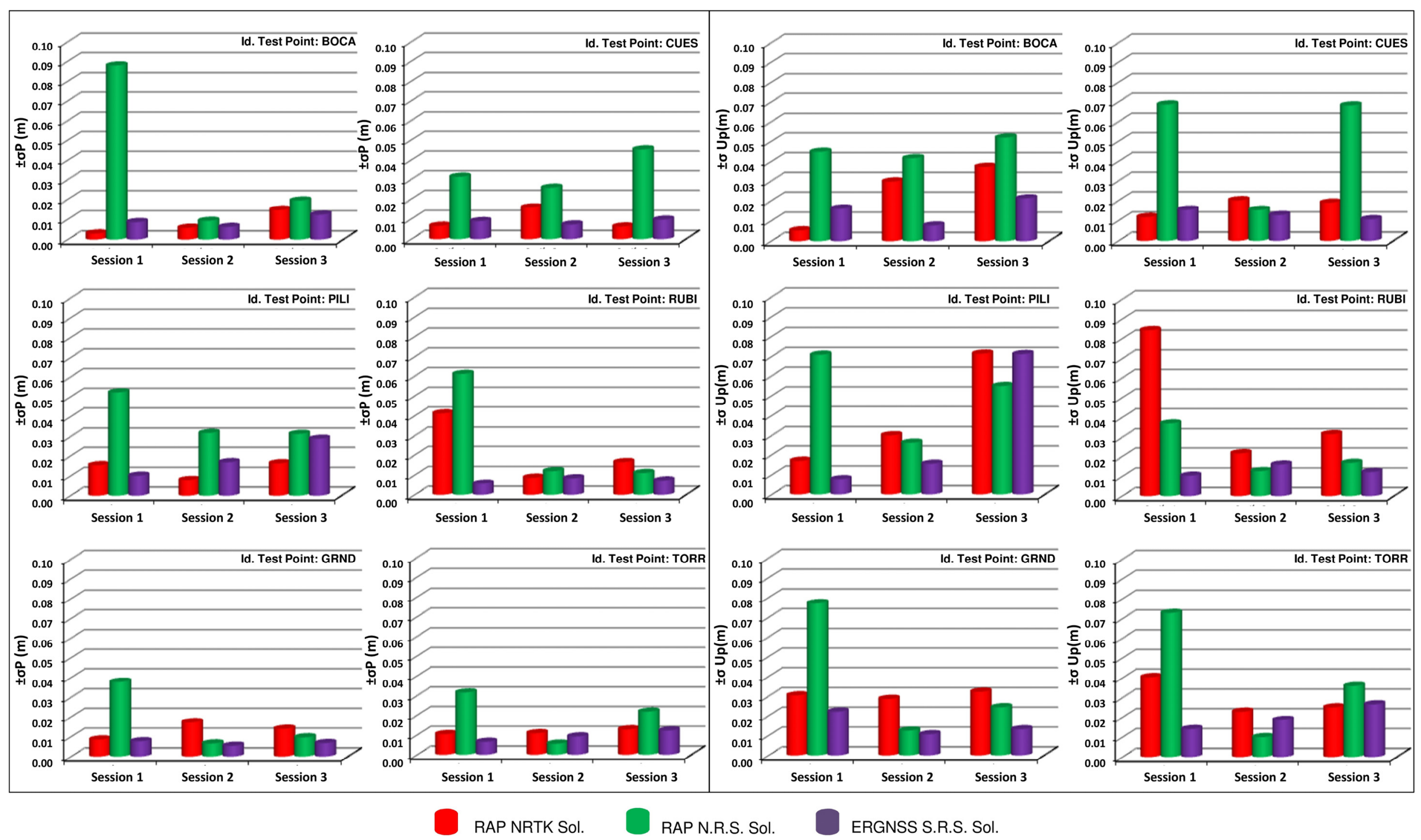

Fig. 4 Planimetric (left) and altimetric (right) precision for the RAP and ERGNSS solutions at test points. 
receivers are essentially identical and the errors common to both receivers tend to be cancelled out. Figure 4 shows the planimetric and altimetric precision by session at the test points. The planimetric precision $(\sigma \mathrm{P})$ is computed as the square root of the sum of variances for the North and East components.

For the RAP NRTK solution, the mean planimetric precision is around a centimeter, as well as considering the ERGNSS solution. However, RAP N.R.S solution achieves a mean value of 3 centimeters. Despite the time period between the three observation sessions, no significant differences in the planimetric results of the three sessions were found, except for the RUBI and BOCA test points considering the RAP NRTK and RAP N.R.S solutions respectively. At the RUBI test point, the review of the reference station used as the "master" station showed no changes throughout the different sessions and was always the nearest station to the test point (CRDB) located less than $10 \mathrm{~km}$ away. However, the user can not know the subset of stations used to compute the network corrections through the different sessions. At the BOCA test point, considering the N.R.S solution, the reference station changes throughout the different observation sessions with baselines ranging from 6 to $46 \mathrm{~km}$. Considering the ERGNSS S.R.S solution the worse precision in the Up component is for the PILI test point (session 3 ) with $0.071 \mathrm{~m}$. For all other test points and in all sessions considered the value of the altimetric precision is better than $0.025 \mathrm{~m}$. Considering the RAP N.R.S solution, the precision values are slightly worse, ranging from $0.010 \mathrm{~m}$ at TORR to $0.077 \mathrm{~m}$ at GRND in session 2. The mean altimetric precision is $0.041 \mathrm{~m}$. For the RAP NRTK solution, the precision values range between $0.005 \mathrm{~m}$ in session 1 at the BOCA test point and 0.084 in the same session at RUBI. This also highlights the precision corresponding to PILI in session 3 with $0.071 \mathrm{~m}$. The mean altimetric precision is $0.031 \mathrm{~m}$.

In order to analyze the repeatability of RTK solutions, multiple observation sessions at each test point are considered. Sessions 1, 2 and 3 are spread throughout the day (morning, mid-day and afternoon) so that the satellite configurations and atmospheric conditions are slightly different. On the graphs in Figure 5 the planimetric (using $10 \times 10 \mathrm{~cm}$ grid) and altimetric RTK positions based on the RAP network registered at GRND test point in session 1, 2 and 3 are plotted. This point was selected because it has large distance and elevation difference from the RAP nearest reference station. Comparing the values of each component we can see, as expected, the dispersion in the Up component is worse than in the planimetric component. The large scatter in the altimetric component shows the susceptibility to tropospheric biases due to height (Song et al., 2014). In contrast, the distance is not as decisive as the height, especially when the rover is located inside the active network. Graphical results of planimetric RTK positioning shown that, for session 1, the two solutions are grouped in a few separate and internally consistent blocks. However, for session 2 and 3 a larger dispersion is shown. Changes in satellite configuration (number of satellites, PDOP, etc.) might be one of the reasons. High HDOP and VDOP values decrease the accuracy of RTK positioning. A review of VDOP values shows that the worst values of the observation period at the GRND test point correspond to the observation session 3 where the minimummaximum values for VDOP are 1.2 - 2.5. For session 1 and 2 the VDOP value ranges between 1.1 and 2.0. If the NRTK solution is considered, the greater dispersion in sessions 2 and 3 can be related to the master reference station used throughout the different observation sessions. While in the first session the master reference station is always the nearest reference station (MLGA located at just $22.4 \mathrm{~km}$ ), in the second and third sessions the master reference stations are MLGA and CABR (the latter is located $65 \mathrm{~km}$ from the GRND test point), although the approximate position sent to the server using the NMEA GGA format is always the same.

An active network provides the reference frame, offering a datum-consistent solution to users. RTK positioning accuracy depends on the reference frame but is also affected by the reference stations' distribution (distance to the reference stations and elevation difference between the reference stations and the test site). The horizontal and vertical accuracy at all test points selected are summarized by session in Table 4. It shows generally more accurate values for the horizontal component than for the vertical component. Horizontal accuracy values reach a maximum value of $0.041 \mathrm{~m}$ for the RAP NRS solution. The value obtained for the ERGNSS S.R.S solution is similar $(0.035 \mathrm{~m})$. When the RAP NRTK solution is considered the horizontal accuracy is better, reaching a maximum value of $0.025 \mathrm{~m}$. If we consider the vertical accuracy, the results are worse. Highlights the high values that the BOCA test point presents throughout the three observation sessions for the ERGNSS S.R.S solution.

Considering the nearest single-reference station solution or the NRTK solution based on the RAP network, the respective values of vertical accuracy are better than 4 centimeters, except for session 3, which also reach $8 \mathrm{~cm}$. Incorrect calculation of the control position for the BOCA test point is discarded as shown in Table 3. The vertical accuracy values exceed $8 \mathrm{~cm}$, but only for one of the three sessions performed. This shows that the RTK accuracy is also affected by the reference station or subset of reference stations considered in each session, although the approximate position of the test site sent to the control center is the same throughout the three sessions. The same occurs in session 2 at the TORR test point.

\section{CONCLUSIONS}

Today real time precise positioning is still linked to the active network concept, and this paper has 


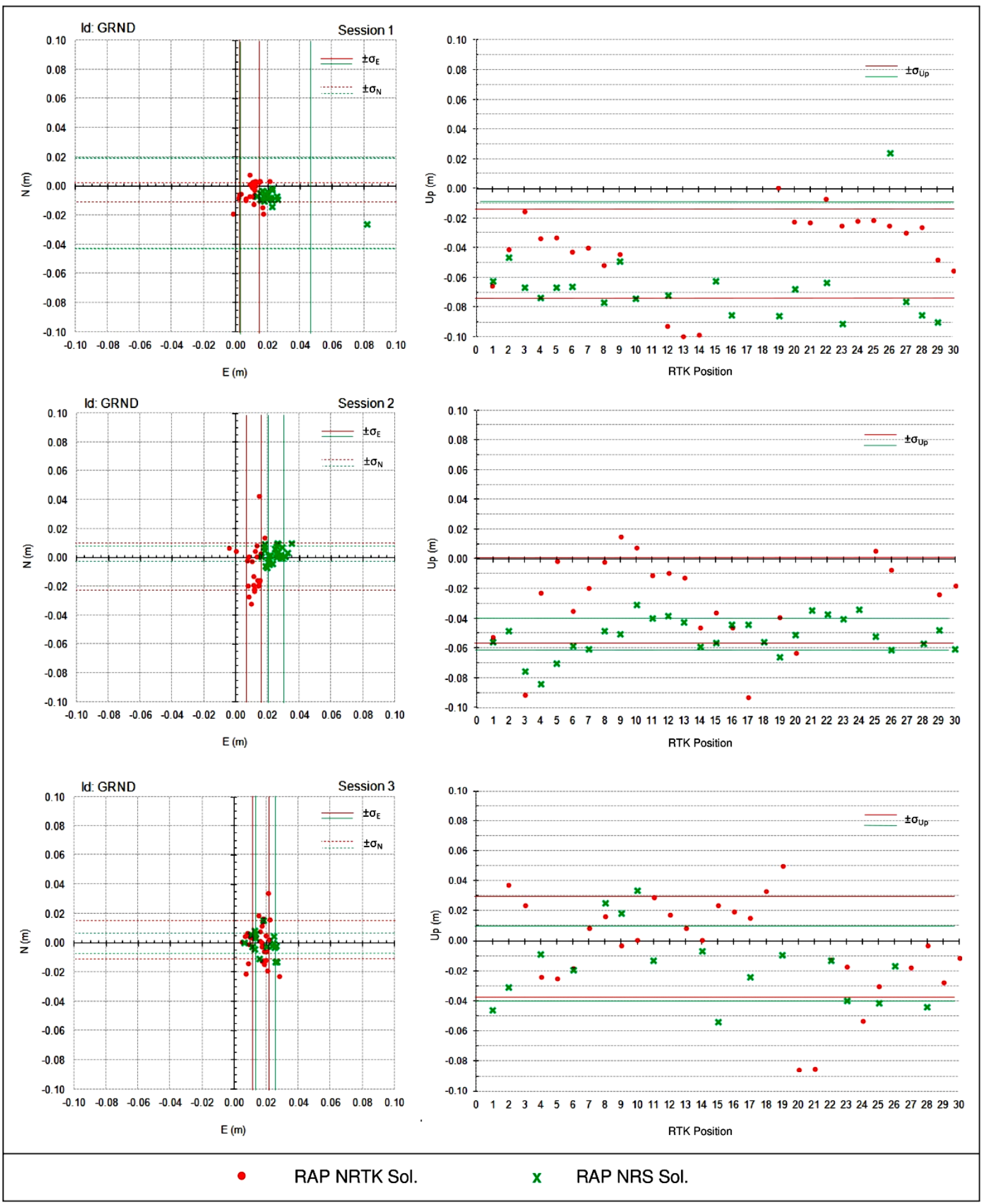

Fig. 5 Graphical results of planimetric (left) and altimetric (right) RTK positioning by session at "GRND" test point considering NRTK and NRS solutions based on the RAP network (control value: adjusted postprocessing solution 
Table 4 Horizontal (H) and vertical (V) accuracy by session at test points considering RAP NRTK, RAP NRS and ERGNSS SRS solutions.

\begin{tabular}{cccccccc}
\hline \multirow{2}{*}{ Id. Test Point } & \multirow{2}{*}{ Session } & \multicolumn{2}{c}{ RAP NRTK } & \multicolumn{2}{c}{ RAP NRS } & \multicolumn{2}{c}{ ERGNSS SRS } \\
\cline { 2 - 7 } & & H(m) & V(m) & H(m) & V(m) & H(m) & V(m) \\
\hline \multirow{2}{*}{ BOCA } & S1 & 0.008 & 0.039 & 0.041 & 0.038 & 0.008 & 0.102 \\
& S2 & 0.009 & 0.037 & 0.010 & 0.032 & 0.007 & 0.105 \\
& S3 & 0.018 & 0.080 & 0.029 & 0.071 & 0.013 & 0.141 \\
\hline \multirow{2}{*}{ CUES } & S1 & 0.016 & 0.036 & 0.013 & 0.050 & 0.009 & 0.013 \\
& S2 & 0.015 & 0.018 & 0.019 & 0.015 & 0.012 & 0.035 \\
& S3 & 0.013 & 0.016 & 0.024 & 0.036 & 0.014 & 0.072 \\
\hline \multirow{2}{*}{ PILI } & S1 & 0.019 & 0.027 & 0.030 & 0.038 & 0.024 & 0.064 \\
& S2 & 0.020 & 0.022 & 0.020 & 0.050 & 0.015 & 0.015 \\
& S3 & 0.025 & 0.080 & 0.019 & 0.063 & 0.035 & 0.072 \\
\hline \multirow{2}{*}{ RUBI } & S1 & 0.022 & 0.041 & 0.032 & 0.021 & 0.007 & 0.047 \\
& S2 & 0.011 & 0.016 & 0.016 & 0.011 & 0.009 & 0.048 \\
& S3 & 0.017 & 0.025 & 0.016 & 0.018 & 0.011 & 0.036 \\
\hline \multirow{2}{*}{ GRND } & S1 & 0.013 & 0.045 & 0.033 & 0.090 & 0.008 & 0.066 \\
& S2 & 0.019 & 0.030 & 0.025 & 0.052 & 0.005 & 0.062 \\
& S3 & 0.019 & 0.025 & 0.019 & 0.026 & 0.007 & 0.013 \\
\hline \multirow{2}{*}{ TORR } & S1 & 0.013 & 0.050 & 0.019 & 0.036 & 0.006 & 0.054 \\
& S2 & 0.019 & 0.081 & 0.009 & 0.015 & 0.012 & 0.026 \\
& S3 & 0.012 & 0.043 & 0.026 & 0.080 & 0.016 & 0.025 \\
\hline
\end{tabular}

highlighted two relevant issues in RTK positioning based on an active network. The first is the importance of knowing the reference frame and reference epoch involved in order to assure the positioning accuracy at centimeter level required for many geomatic purposes. In this aspect the networks analyzed should follow the recommendation of EUREF Technical Working Group (TWG) and expressing the ETRS89 coordinates in the ETRF2000 which is the conventional frame of the ETRS89 (Bruyninx et al., 2013). Second, the specific numerical results of precision for each test site reveal that the quality values (CQ) provided by the rover equipment are often very optimistic and do not represent the real precision of the RTK solutions, both in the planimetric and altimetric solutions. These values should therefore be used with caution by the users of a RTK network.

In order to analyze the performance of RTK positioning based on a national and a local active network in southern Spain, we selected a representative sample of geodetic sites as test points. The main quality parameters considered in RTK positioning have been: TTFA, precision, accuracy and repeatability. Statistical results from real time data collected and compared to post-processing solutions allowed us to evaluate the performance of ERGNSS and RAP networks. Considering the TTFA, the highest percentages of registered positions whose time to fix ambiguities is greater than 90 seconds are for test points located on the southern border of Andalusia where the NRTK solutions are extrapolated. The ERGNSS SRS solution has the highest percentage of registered positions in automatic mode. GLONASS can be used in an integrated approach with GPS satellites in order to increase the accuracy and speed of the ambiguity resolution. With the ERGNSS SRS solution in the area of Andalusia we have confirmed that the precision in the East, North and Up components attainable is within 3-19 mm, 4-22 mm and $8-71 \mathrm{~mm}$ respectively considering all the observation sessions. If the RAP NRS solution is considered, these values are within 4-88 mm, 4$60 \mathrm{~mm}$ and 10-77 $\mathrm{mm}$ respectively. For the RAP NRTK solution the precision is within 2-26 $\mathrm{mm}, 2-$ $2 \mathrm{~mm}$ and 5-84 $\mathrm{mm}$ for the East, North and Up components. In general, the planimetric NRTK solutions, mainly affected by the distance to the nearest reference station and the subset of reference stations considered, is more precise and accurate that the altimetric solution. The horizontal accuracy value is better than $0.025 \mathrm{~m}$ for the RAP NRTK solution and better than $0.041 \mathrm{~m}$ for the RAP NRS solution. A medium value was obtained for the ERGNSS S.R.S solution. These values are duplicated and sometimes triplicated for vertical accuracy. The numerical results throughout the different sessions confirm that minor changes in satellite configuration and atmospheric conditions slightly affect precision and accuracy in RTK positioning. In the southern part of the Andalusian Community the precision results show that the most significant errors impact on the Up component. The addition of GLONASS observables in the RAP reference stations could reduce the error in this component. As shown in Emardson et al. (2009) the inclusion of other satellite systems can reduce the error in the vertical position.

Currently the ERGNSS network has a more prominent role than the classical passive geodetic infrastructure in Spain, not only in reference frame determination but also in many applications of realtime positioning. RAP, as local active network, 
constitutes an essential positioning infrastructure for surveying, mapping and all kinds of geomatic applications in the Andalusian Community (S Spain). This network complements the precise positioning services provided by the ERGNSS network, a Spanish active network, ensuring accurate real time positioning, full coverage and reliable positioning services in the region. Considering the reduced number of reference station that ERGNSS network has in the Andalusian Community, the statistical results confirm that it is possible to achieve centimeter-level accuracy with ERGNSS RTK positioning for baselines up to $20 \mathrm{~km}$ length. The results are similar to those achieved with the RAP N.R.S solution and improve slightly with the RAP NRTK solution.

In order to improve the reliability of RTK positioning in the Andalusian Community, currently the RAP network is immersed in an update process. The IGNE (ERGNSS network) and IECA (RAP network) have started a collaboration process whose objectives are the integration of both networks in the community of Andalusia and its closest environment, the increase of accurate real time positioning services including new NRTK solutions and the optimization of economic resources in geodetic infrastructure in the region. As shows Higgins (2008) the actions on active networks should be aimed at limiting infrastructures duplication, improving the performance and efficiency of positioning services. In other Spanish regions, such as the Valencian Community (Capilla et al., 2006) or more recently in the Balearic Islands (Sánchez-Alzola et al., 2014) with their respective active networks ERVA and XGAIB, the integration of reference stations belonging to local networks and ERGNSS reference stations to improve the efficiency of real time positioning services is a proven fact.

\section{ACKNOWLEDGEMENT}

Support provided by the Institute of Statistics and Cartography of Andalusia and Spanish National Geographic Institute is gratefully acknowledged. This research was financed by the University of Jaén in collaboration with “Caja Rural Jaén” (UJA2015/06/11 Project) and RNM282 - Microgeodesia Jaén Research Group (Junta de Andalucía). Figure 1 was realized using Generic Mapping Tools (Wessel and Smith, 1998). We appreciate the valuable comments of the reviewers, which contributed to the improvement of this paper.

\section{REFERENCES}

Afonso, A., Teodoro, R. and Mendes, V: 2008, SERVIR the Portuguese Army CORS Network for RTK, Integrating Generations, FIG Working Week 2008, Stockholm, Sweden, 14-19 June.

Bakula, M.: 2013, Study of reliable rapid and ultrarapid static GNSS surveying for determination of the coordinates of control points in obstructed conditions. Journal of Surveying Engineering, 139, 188-193. DOI:10.1061/(ASCE)SU.1943-5428.0000109
Benciolini, B., Biagi, M., Crespi, M., Manzino, A.M. and Roggero, M.: 2008, Reference frames for GNSS positioning services: Some problems and proposed solutions. Journal of Applied Geodesy, 2, 53-62. DOI: 10.1515/JAG.2008.006

Boriskin, A., Kozlov, D. and Zyryanov, G.: 2012, The RTCM multiple signal messages: A new step in GNSS data standardization. Proceedings of the 25th International Technical Meeting of The Satellite Division of the Institute of Navigation (ION GNSS 2012), Nashville, TN, 2947-2955.

Boucher, C. and Altamimi, Z.: 2011, Memo: Specifications for reference frame fixing in the analysis of a EUREF GPS campaign (http://etrs89.ensg.ign.fr/memo-V8.pdf)

Brockmann, E.: 2016, Official national ETRF coordinates for EPN stations. EUREF symposium, San Sebastian, Spain

(http://www.euref.eu/documentation/Tutorial2016/t06-Brockmann.pdf)

Bruyninx, C., Altamimi, Z., Caporali, A., Kenyeres, A., Lidberg, M., Stangl, G. and Torres, J.A.: 2013, Guidelines for EUREF Densifications (previously know as "EUREF Campaign Guidelines") (http://www.epncb.oma.be/ documentation/guidelines/)

Bruyninx C.: 2007, Comparing GPS-only with GPS + GLONASS positioning in a regional permanent GNSS network. GPS Solutions, 11, 2, 97-106. DOI: 10.1007/s10291-006-0041-9

Capilla, R.M., Die, J. and Esteso, E.: 2006, Design, integrated applications and performance of the active GPS/GNSS network of Valencia. 7 Setmana Geomàtica. Barcelona, Spain, (In Spanish).

Dettmering, D. and Webber, G.: 2004, The EUREF-IP Ntrip Broadcaster: Real-time GNSS data for Europe. Proceedings of the IGS2004 Workshop. Astronomical Insitute, University of Bern, Switzerland. March 1-5. (http://www.igs.org/assets/archive/04_rtberne/cdrom/ Session3/3 4 Dettmering.pdf)

Edwards, S.J., Clarke, P.J., Penna N. T. and Goebell, S.: 2010, An examination of network RTK GPS services in Great Britain, Survey Review, 42, 316, 107-121. DOI: 10.1179/003962610X12572516251529

Emardson, T., Jarlemark, P., Bergstrand, S., Nilsson, T. and Johansson, J.: 2009, Measurement Accuracy in Network-RTK. SP Report 2009:23. SP Technical Research Institute of Sweden, Borås.

Euler, H-J., Keenan, C.R., Zebhauser, B.E. and Wübbena, G.: 2001, Study of a simplified approach in utilizing information from permanent reference station arrays. 14th Int. Tech. Meeting of the Satellite Division of the U.S. Institute of Navigation, Salt Lake City, Utah, 379-391.

Feng, Y. and Wang, J.: 2008, GPS RTK performance characteristics and analysis. Journal of Global Positioning Systems, 7, 1, 1-9.

Higgins, M.B.: 2008, An organisational model for a unified GNSS reference station network for Australia. Journal of Spatial Science, 53, 2, 81-95. DOI: $10.1080 / 14498596.2008 .9635151$

Kenyeres, A.: 2009, Maintenance of the EPN ETRS89 coordinates. EUREF TWG 2009 Spring Meeting, Budapest,

(http://www.euref.eu/TWG/EUREF\%20TWG\%20min utes/49-Budapest2009/03-eETRSmaint TWGrepBP.pdf) 
Lei, Q., Lei, L. and Zemin, W.: 2010, An tropospheric delay model for GPS NET RTK. Proc. 2010 2nd Int. Conf. Inf. Technol. Comput. Sci., July 2010, 98-101. DOI: 10.1109/ITCS.2010.30

Páez, R., Torrecillas, C., Barbero, I. and Berrocoso, M.: 2017, Regional positioning services as economic and construction activity indicators: the case study of Andalusian Positioning Network (Southern Spain). Geocarto International, 32, 1, 44-58.

DOI: $10.1080 / 10106049.2015 .1120358$

Rizos, C.: 2007, Alternatives to current GPS-RTK services and some implications for CORS infrastructure and operations. GPS Solutions, 1, 3, 151-158. DOI: 10.1007/s10291-007-0056-x

Sánchez-Alzola, A., Sánchez, C., Giménez, J., Alfaro, P., Gelabert, B., Borque, M.J. and Gil, A.J.: 2014, Crustal velocity and strain rate fields in the Balearic Islands based on continuous GPS time series from the XGAIB network (2010-2013). Journal of Geodynamics, 82, 78-86, DOI: $10.1016 / j . j 0 g .2014 .05 .005$

Schwieger, V., Lilje, M. and Sarib, R.: 2009, GNSS CORS Reference frames and services. 7th FIG Regional Conference Spatial Data Serving People: Land Governance and the Environment - Building the Capacity. Hanoi, Vietnam.

Seeber, G.: 2003, Satellite geodesy: foundations, methods, and applications. Walter de Gruyter, Berlin, New York.

Song, J., Kee, C., Park, B., Park, H. and Seo, S.: 2014, Correction combination of compact network RTK considering Tropospheric Delay Variation over height. Proceedings of IEEE/ION PLANS 2014, Monterey, CA, May. DOI: 10.1109/PLANS.2014.6851362

Spanish Committee of Geodesy and Geophysics: 2011, National Report on Geodesy for 2007-2010. XXV General Assembly of the International Union of Geodesy and Geophysics. Melbourne, Australia.
Takac, F. and Zelzer, O.: 2008, The relationship between network RTK solutions MAC, VRS, PRS, FKP and iMAX. Proceedings of ION GNSS 2008, Savannah, GA.

Tsakiri, M.: 2011, Evaluation of GPS/Galileo RTK network configuration: Case study in Greece. Journal of Surveying Engineering, 137, 4, 156-166.

DOI: 10.1061/(ASCE)SU.1943-5428.0000054

Wang, C., Feng, Y., Higgins, M. and Cowie, B.: 2010, Assessment of commercial network RTK user positioning performance over long inter-station distances. Journal of Global Positioning Systems, 9, 1, 78-89.

Weber, G., Gebard, H. and Dettmering, D.: 2005, Networked Transport of RTCM via Internet Protocol (NTRIP). In: A Window on the Future of Geodesy, Proceedings of the International Association of Geodesy. IAG General Assembly, Sapporo, Japan. Series: International Association of Geodesy Symposia, 128, Sanso, F. (Ed.). DOI: $10.1007 / 3-540-27432-411$

Wegener, V. and Wanninger, L.: 2005, Communication options for Network RTK / SAPOS ${ }^{\circledR}$ realization. Joint 2nd Workshop on Positioning, Navigation and Communication 2005 (WPNC'05) \& 1st UltraWideband Expert Talk 2005 (UET`05), Hannover, Germany.

Wessel, P. and Smith, W.H.F.: 1998, New improved version of the generic mapping tools released. EOS Trans. AGU, 79, 579. DOI: 10.1029/98EO00426 